\title{
Феномен присутствия при решении интеллектуальных задач: результаты качественного исследования
}

\author{
Н.В. Авербух \\ Уральский Федеральный Университет \\ natalya_averbukh@mail.ru
}

\section{Аннотация}

Данная статья посвящена проблеме изучению феномена присутствия при решении интеллектуальных задач. В качестве интеллектуальной задачи выбран субтест текста Векслера «Кубики Коса». Феномен присутствия исследуется с помощью структурированного контекстного интервью.

В данной статье ставится вопрос о возможности исследования присутствия при решении интеллектуальной задачи в предназначенной для этого виртуальной среде, в связи с этим бедной объектами и событиями.

В рамках результатов показывается, что феномен присутствия может сочетаться как с успешным решением интеллектуальной задачи, так и с неспособностью её решить. Показывается, как при решении переживаются пространственное и социальное присутствие.

Ключевые слова: виртуальная реальность, феномен присутствия, пространственное присутствие, социальное присутствие, тест «Кубики Коса»

Библиографическая ссылка: Авербух Н.В. Феномен присутствия при решении интеллектуальных задач: результаты качественного исследования // Информационное общество: образование, наука, культура и технологии будущего. Выпуск 3 (Труды XXII Международной объединенной научной конференции «Интернет и современное общество», IMS-2019, Санкт-Петербург, 19 - 22 июня 2019 г. Сборник научных трудов). - СПб: Университет ИТМО, 2019. С. 211-225. DOI: 10.17586/2587-85572019-3-211-225

\section{1. Постановка задачи}

Виртуальная реальность применяется для разнообразных целей, например, в качестве авиационных тренажёров, очень активно используется в играх, а также для целей компьютерной визуализации - зримого представления компьютерной моделей. Одной из составляющих компьютерной визуализации является научная визуализация, в частности, связанная с отображением результатов математического моделирования. Виртуальная среда, созданная для научной визуализации, отличается тем, что предназначена для целей умственной работы и решения задач высокой степени абстракции. Эффективность решения этих задач не в последнюю очередь зависит от умения увидеть неочевидную с первого взгляда взаимосвязь между объектами или явлениями. Психологическое изучение человека, взаимодействующего со средой, предназначенной для научной визуализации, должно строиться как на исследовании специфических процессов для виртуальной реальности, так и на моделировании умственной деятельности специалиста.

С точки зрения психологии центральным понятием для виртуальной реальности является понятие феномена присутствия. Это многогранный феномен, которому 
посвящено множество работ, в частности, сборник статей, содержащий обширные материалы по вопросу присутствия [8].

В центре внимания данной статьи будут две разновидности феномена присутствия это так называемое пространственное и социальное [8]. В настоящей работе под пространственным присутствием понимается переживание, в ходе которого человек ощущает себя внутри виртуального пространства, воспринимая его как реальное. Делается предположение, что пространственное присутствие может не только ощущаться не всё время на протяжении взаимодействия с виртуальной средой, но и переживаться частично: та ситуация, когда человек сообщает, что он был, например, «вроде бы там, но и не там». Социальное присутствие означает ощущение пребывания рядом другого человека или совместное пребывание в одном пространстве (так называемое со-присутствие). В данной статье рассматривается социальное присутствие как ощущение рядом другого человека.

Из относительно недавних работ в связи с вопросами пространственного и социального присутствия, а также методов их измерения следует упомянуть [10], в которой изучается поведенческий аспект пространственного присутствия, которое констатируется, когда пользователи ведут себя в виртуальной среде аналогично тому, как ведут себя в реальности. В работе [22] ставится вопрос о том, что эмоциональное погружение может больше, чем пространственное влиять на присутствие и такие его параметры, как искажение восприятия времени, реализма, сенсорных параметров и т. п. В работе [11] испытуемым предлагается заполнять анкеты в виртуальной среде, так как выход из виртуальной среды для заполнения анкет может вызвать дезориентацию. В работе [12] ставится вопрос о реализме и достоверности виртуальной среды и происходящего в ней со ссылкой на работы группы М. Слейтера (M. Slater). Кроме того, в этой работе говорится о социальном присутствии и со-присутствии как о восприятии других людей рядом и способности быть воспринятыми ими. Близкие вопросы поднимаются в [9], входящей в сборник [8], о перцептивном и социальном реализме. Перцептивный реализм связан с поддержкой средой действий пользователе в нём. Социальный реализм связан с правдоподобием. Также в этой работе говорится о пространственном и социальном присутствии, понимаемом и как совместное присутствие и как ощущение присутствия других людей рядом.

Отдельно стоит рассмотреть работы группы М. Слейтера (M. Slater). Сравнивая состояние присутствие с тем, как в предложенных гештальтистами иллюзиях восприятие перемещается между фигурой и фоном и делается сенсорный выбор (так и в виртуальной реальности делается выбор между виртуальной и реальной средой), [14], он предлагает исследовать прерывания присутствия (Breaks in Presence, BIP) [15], то есть ситуации, когда у пользователя, находящегося в виртуальной реальности, пропадает ощущение, что происходящее вокруг него реально и он вспоминает, что он находится в реальном помещении. В большой работе [16] подробно описываются возможности измерения присутствия при помощи подсчёта прерываний, о которых испытуемый инструктируется сообщать во время пребывания в виртуальной среде. В дальнейших экспериментах прерывания запускались искусственно, [7] в виде перекрывающего изображение белого шума. Кроме этого, большой интерес представляет введённая коллективом Слейтера концепция иллюзии места, которой он предлагает заменить перегруженное разными смыслами понятие присутствия, и понятие правдоподобия (происходящего в виртуальной среде) [13]. В последние годы исследовательские интересы коллектива Слейтера связаны с концепцией иммерсивной журналистики, где человеку с помощью средств виртуальной реальности предлагается пережить остросоциальные события [17].

Следует отметить такие отечественные работы как [3-5], посвящённые когнитивному контролю и метакогнитивным процессам и их влиянию на переживание чувства присутствия. В дальнейшем эти исследования были продолжены в работах [18-20].

Виртуальные среды, созданные для игровых целей, можно назвать активными, насыщенными событиями средами. С участником игры постоянно что-то происходит - 
само по себе или в ответ на его действия. Среды, предназначенные для научных целей, значительно более пассивны. Даже если представленные там объекты обладают динамикой, с участником среды не происходит событий. Он наблюдает, вносит свои изменения и делает выводы.

Данная работа является логическим продолжением работ [1] и [2]. В этих работах описывается разработанный для целей изучения присутствия метод структурированного контекстного интервью, в рамках которого испытуемым задавали вопросы относительно произошедших в виртуальной среде событий. Метод интервью для исследования присутствия, в том числе социального, описан ещё в работе [6] в 2004 году. Коллектив Слейтера также в 2004 году в работе [7] использовал интервью, но не для изучения переживания присутствия как такового, а для изучения переживаний во время прерываний в присутствии (BIP).

В работах [1] и [2] упоминается активная среда, наполненная событиями, на которые испытуемые могли реагировать как на события реального мира или как на виртуальные, нереальные ситуации. Однако в пассивной среде не происходит событий. Пользователь не может сказать «когда я чуть не налетел на дерево, я испытал страх», не может сообщить об ожидании ветра в лицо. В пассивной среде происходит только то, что делает с ней сам пользователь. Это усложняет задачу исследования присутствия при помощи контекстного интервью. Это ставит вопрос о том, возможно ли присутствие как таковое в пассивной среде.

Задача исследования присутствия при решении интеллектуальной задачи ставилась в работе [2]. В настоящей работе воссоздан эксперимент, описанный в [2] и проведено исследование присутствия методом контекстного интервью. За основу взята та же интеллектуальная задача, субтест теста Векслера, «Кубики Коса». Использованная для нужд эксперимента модификация в полной мере требует от исполнителя умения увидеть неочевидную на первый взгляд взаимосвязь между объектами (см. раздел 2.2), поэтому решение данной задачи до некоторой степени моделирует деятельность специалиста, взаимодействующего с системой научной визуализации.

Как уже говорилось выше, в центре внимания в настоящей работе находится пространственное присутствие, касающееся переживания присутствия в виртуальном пространстве, и социальное присутствие.

В классической работе [21] в опросник, посвящённый присутствию, входит утрата связи с окружающим миром: игнорирование отвлекающих факторов, незаметность хода времени и т. п. Эти явления вполне могут происходить и при обычном сосредоточении, которое может указывать на вовлечённость [8], но не на присутствие в виртуальной реальности. Мало не обращать внимания на шум, нужно ещё ощущать себя находящимся в виртуальной среде. В ходе работы будет показано, что игнорирование внешних шумов не является обязательной составляющей переживания феномена присутствия.

В работе [1] было показано, что за частью переживания феномена присутствия может стоять не реальный контакт с объектами, а только ожидание (тот же самый ветер в лицо или, например, удар ветками). В среде, представляющую собой комнату, в которой стоит стол, на котором испытуемый складывает требуемый узор из верхних граней кубиков, не представляется адекватным ожидание каких-либо событий. Относительно близким аналогом можно признать вопрос о желании исследовать среду, а также вопрос о желании передвигать кубики непосредственно руками, игнорируя предложенный контроллер. Следует отметить, что сам по себе интерес к среде не является определяющим признаком присутствия, так как интерес может вызвать и нарисованная комната. В то же время желание взглянуть на свои руки, держащие контроллер, может считаться признаком того, что человек не испытывает присутствие: он помнит о средстве управления.

Таким образом, в данной работе за составляющие переживания пространственного присутствия были выбраны следующие компоненты:

— игнорирование окружающей реальности; 
— интерес к представленной среде;

— «забывание» о средстве управления;

- желание вступить в физическое взаимодействие с объектами виртуальной среды.

Кроме этого, испытуемым задавался прямой вопрос, ощущали ли они ту среду реальной или чувствовали ли они себя находящимися «там», в представленной комнате. Поскольку присутствие является субъективным переживанием, решение, было ли оно или не было, так или иначе следует оставить за самим переживающим его человеком, во всех остальных случаях говоря только о переживании его элементов.

Что касается социального присутствия, то здесь, в отличие от работы [1], речь не идёт о совместном присутствии в виртуальной среде. При работе с системами научной визуализации на базе виртуальной реальности обычно практикуется индивидуальная, а не групповая работа (хотя, и она при необходимости возможна), однако не исключается присутствие в помещении другого человека. Возможно, коллеги, который занимается своими исследованиями, возможно, разработчика системы, возможно, участника общего проекта, который ждёт своей очереди на взаимодействие с системой. Вариантов может быть множество. Чем будет являться присутствие другого в том реальном помещении, откуда пользователь выходит в виртуальную среду? Частью реального мира, о котором забывают в пылу работы? Или участником взаимодействия? В работе [1] показано, как в представлениях одного и того же человека сочетается нахождение другого человека рядом с ним в комнате - и собственное нахождение в виртуальном вертолёте. Возможно ли это при взаимодействии с пассивной средой, при решении интеллектуальной задачи?

Итак, в рамках данной работы социальное присутствие исследуется посредством вопросов «Где была экспериментатор?», «Насколько чётко ощущалось её местонахождение в пространстве?» и т. п.

\section{2. Методика}

\section{1. Испытуемые}

В исследовании принимали участие 16 человек, 8 мужчин и 8 женщин в возрасте от 19 до 38 лет. 6 человек (1 мужчина и 5 женщин) имели высшее образование, 1 женщина учёную степень, 7 человек (5 мужчин и 2 женщины) - неоконченное высшее образование и 2 человека (мужчина и женщина) - среднее специальное образование. Большинство испытуемых являются опытными пользователями компьютера, за исключением 1 женщины (начинающий пользователь), 4 человека (3 мужчины и 1 женщина) профессионально работают с компьютерами: дизайнер, системный администратор, программист, студент, изучающий компьютерные науки. Среди испытуемых только у одного не было никакого опыта взаимодействия с 3D-системами (мужчина), у одного был опыт взаимодействия с очками виртуальной реальности (мужчина), у 7 (2 мужчины и 5 женщин) - опыт просмотра 3D-кино, у 6 (3 мужчины и 3 женщины) опыт взаимодействия с очками виртуальной реальности и просмотра 3D-кино, у 1 мужчины - опыт

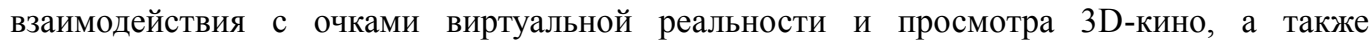
знакомство с системой виртуальной реальности типа CAVE.

\section{2. Оборудование}

В эксперименте использовалась программная система, созданная на базе межплатформенной среды разработки компьютерных игр Unity. В качестве контроллера предлагался геймпад Defender Game Racer RS3. В качестве средства вывода использовались стереоскопические очки Oculus Rift 2 с настроенным разрешением $1920 * 1080$.

Программа состояла из трёх частей: интерактивная инструкция, позволяющая освоить управление виртуальным кубиком с помощью геймпада, тренировочная серия, в ходе 
которой испытуемым предлагалось сложить узор из внешних граней разноцветных кубиков по представленному справа образцу, и основная серия, состоящая из 10 заданий, соответствующих заданиям теста «кубики Коса».

Виртуальная среда представляла из себя комнату, в которой находился стол, на котором справа на куске клеёнки лежала виртуальная карточка с образцом. На всех заданиях границы узора обозначались тонкими красными линиями. На первых девяти заданиях границы карточки совпадали с границами узора. На десятом задании, так как карточка была квадратная, а узор имел форму ромба, границы узора были чётко видны на белом фоне карточки. Поле, на которое было нужно ставить кубики, находилось слева и также обозначалось куском клеёнки, постеленной поверх скатерти. Кубики появлялись по одному, в ближнем к испытуемому левом углу и испытуемый получал возможность, захватив их ловушкой (состоящий из одних рёбер куб), совершать с кубиком необходимые манипуляции: передвигать, поднимать в воздух и опускать, и поворачивать. Как только кубик ставился на стол и ловушка освобождалась, появлялся новый кубик и так, пока не исчерпывался лимит (первые 6 заданий -4 кубика, остальные -9 кубиков). Таким образом, испытуемый не знал заранее, сколько кубиков ему будет предложено в задании, что повторяет условия, описанные в статье [2]. Все кубики можно было ставить только параллельно краю стола. Последнее задание отличалось повышенной сложностью из-за того, что образец был развёрнут на $45^{\circ}$ относительно края стола, а кубики требовалось ставить параллельно краю, и, таким образом, при выстраивании рисунка из верхних граней кубиков от испытуемых всё время требовалось мысленно поворачивать образец.

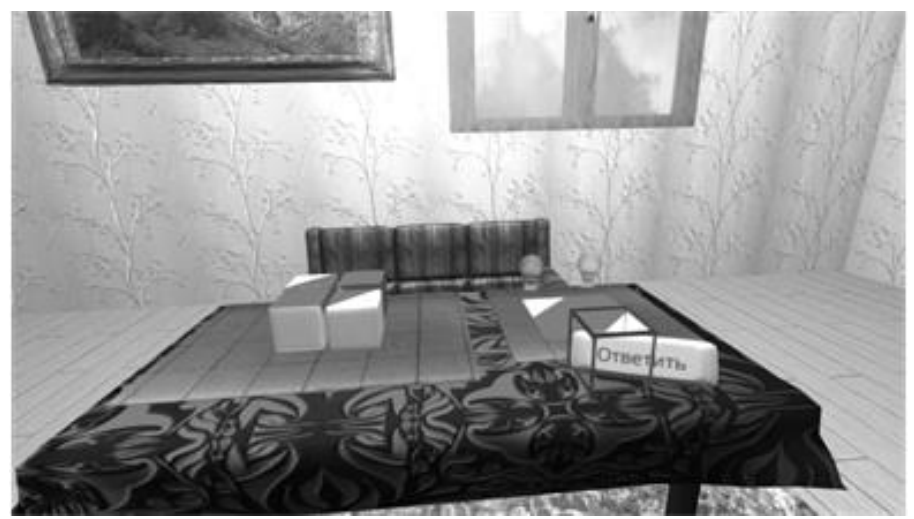

Рис. 1. Пример второго задания

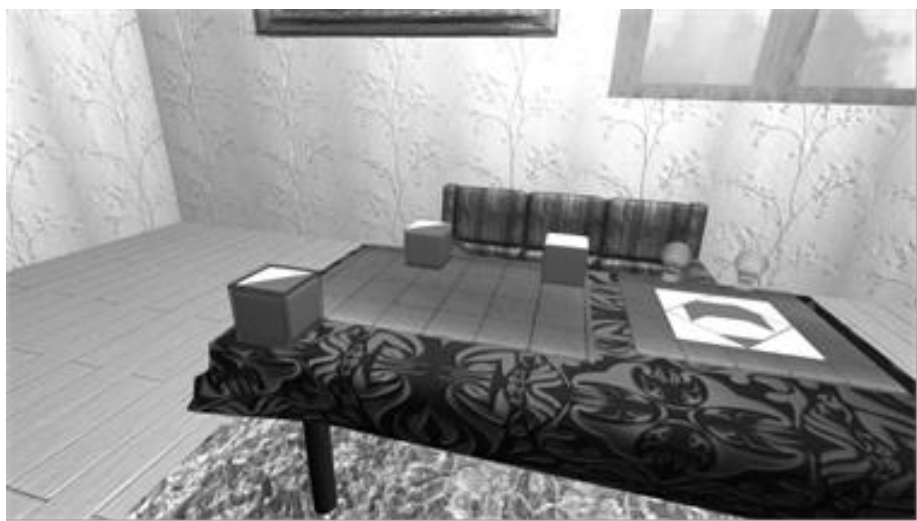

Рис. 2. Пример десятого задания 
Дополнительным затруднением было то, что цвет фона карточки в 10м задании совпадал с цветом части узора (белый), а цвет границы узора совпадал с другим цветом узора (красный), таким образом побуждая испытуемых строить теории относительно их назначения и включать фон в узор. См. рис. 1 и 2.

\section{3. Процедура}

Испытуемым предлагалось заполнить небольшую анкету, после чего перейти сначала к инструкции, потом к тренировочной серии и затем к основной. В ходе инструкции и тренировочной серии при необходимости экспериментатором давались необходимые пояснения. В ходе основной серии испытуемых просили воздержаться от обращений к экспериментатору. Время и правильность выполнения заданий не фиксировались в ходе тренировочной серии и фиксировались в ходе основной. Последнее задание отличалось повышенной сложностью за счёт повёрнутого образца.

По окончанию теста испытуемых в свободной форме опрашивали о переживании ими пространственного и социального присутствия.

Вот примерный список вопросов:

1. Ваши впечатления?

2. Было ли желание исследовать среду?

3. Было ли желание раздвинуть кубики руками?

4. Было ли ощущение, что испытуемый (-ая) находится там, в той среде?

5. Помнил(-а) ли испытуемый (-ая) о присутствии рядом экспериментатора?

6. Слышал(-а) ли испытуемый (-ая) какие-либо посторонние звуки?

При этом вопросы уточнялись по ходу интервью и могли быть внесены в общий план. Так, например, вопросы 2 и 3 возникли из ответов второй и третьей испытуемых о своих впечатлениях.

\section{3. Результаты и обсуждения}

\section{1. Результаты тестирования испытуемых тестом «Кубики Коса»}

Правильность. Первые девять заданий были решены без ошибок всеми испытуемыми. Десятое задание было решено без ошибок одиннадцатью испытуемыми и пятеро не сумели справиться с его решением, отказавшись от него после нескольких неудачных попыток.

Время решения. Для каждого испытуемого было рассчитано суммарное время решения первых девяти заданий. Далее ставился вопрос, есть ли отличия между результатами испытуемых, решившими последнее, десятое задание, и не решившими. Для ответа на этот вопрос был рассчитан коэффициент Манна-Уитни по времени решения первых девяти заданий. Время решения десятого задания не было включено в расчёт, так как для пяти нерешивших это время, скорее, признания отсутствия понимания задания. По результатам расчёта коэффицента Манна-Уитни $\left(\mathrm{U}=21 ; \mathrm{U}_{\text {крит }}=12 ; \mathrm{n}_{1}=11 ; \mathrm{n}_{2}=5\right)$, различия по времени решения первых девяти заданий между решившими и не решившими десятое задание не являются достоверными. Поэтому было рассчитано среднее значение суммарного времени решения первых девяти заданий по всей выборке $\left(\mathrm{t}_{\mathrm{cp}}=1487,38\right.$ сек; $\mathrm{n}$ $=16)$ и для каждого испытуемого - отклонение от среднего.

Время решения последнего задания рассчитывалось только для решивших его. Кроме этого, было рассчитано также среднее время решения последнего задания по выборке решивших, $\left(\mathrm{t}_{\mathrm{cp}}=690,00\right.$ сек; $\left.\mathrm{n}=11\right)$, и для каждого испытуемого рассчитано отклонение от среднего. Далее было высказано предположение, что само по себе время выполнения задания не даёт достаточно информации, так как оно может зависеть не только от того, насколько быстро человек пришёл к решению, но и от того, насколько умело он освоил интерфейс и предложенный контроллер. Поэтому для решивших десятое задание также была рассчитана доля времени решения десятого задания в суммарном времени решения 
всего теста, также среднее значение по группе решивших доли решения последнего задания $\left(\mathrm{d}_{\mathrm{cp}}=31 \%, \mathrm{n}=11\right)$ и отклонение доли решения от среднего.

Результаты расчётов представлены ниже в таблице 1. Испытуемые представлены в порядке возрастания времени решения последнего задания (первые 11), а также возрастания суммарного времени решения первых девяти заданий (для тех, кто не справился с десятым).

Таблица 1. Время решения десятого задания, доля времени решения десятого задания в суммарном времени решения теста, суммарное время решения первых девяти заданий и отклонения от среднего

\begin{tabular}{|l|l|l|l|l|l|l|}
\hline $\begin{array}{c}\text { № } \\
\text { п/п }\end{array}$ & $\begin{array}{c}\text { Время } \\
\text { решения } \\
\mathbf{1 0 г о} \\
\text { задания, } \\
\text { сек }\end{array}$ & $\begin{array}{c}\text { Отклонение } \\
\text { времени } \\
\text { от среднего 10го } \\
\text { времени } \\
\text { решения 10го } \\
\text { задания, сек }\end{array}$ & $\begin{array}{c}\text { Доля времени } \\
\text { решения 10го } \\
\text { задания в } \\
\text { временом } \\
\text { решения } \\
\text { теста, \% }\end{array}$ & $\begin{array}{c}\text { Отклонение } \\
\text { доли } \\
\text { времени } \\
\text { решения } \\
\text { 10го } \\
\text { задания от } \\
\text { среднего, \% }\end{array}$ & $\begin{array}{c}\text { Суммарное } \\
\text { время } \\
\text { решения } \\
\text { первых } \\
\text { девяти } \\
\text { заданий, сек }\end{array}$ & $\begin{array}{c}\text { Отклонение } \\
\text { суммарного } \\
\text { времени } \\
\text { решения } \\
\text { первых девяти } \\
\text { заданий от } \\
\text { среднего, сек }\end{array}$ \\
\hline 1 & 374,28 & 315,72 & 22 & 9 & 1326,52 & 160,86 \\
\hline 2 & 433,28 & 256,72 & 24 & 7 & 1356,59 & 130,79 \\
\hline 3 & 520,26 & 169,74 & 25 & 5 & 1527,46 & $-40,08$ \\
\hline 4 & 578,38 & 111,63 & 33 & -2 & 1199,08 & 288,30 \\
\hline 5 & 579,89 & 110,12 & 26 & 4 & 1618,34 & $-130,96$ \\
\hline 6 & 645,09 & 44,91 & 35 & -5 & 1172,76 & 314,62 \\
\hline 7 & 710,07 & $-20,06$ & 35 & -4 & 1298,68 & 188,70 \\
\hline 8 & 859,78 & $-169,77$ & 38 & -7 & 1429,57 & 57,80 \\
\hline 9 & 884,80 & $-194,80$ & 29 & 2 & 2148,12 & $-660,74$ \\
\hline 10 & 976,72 & $-286,71$ & 36 & -6 & 1706,89 & $-219,51$ \\
\hline 11 & 1027,50 & $-337,49$ & 35 & -4 & 1895,93 & $-408,55$ \\
\hline
\end{tabular}

Таблица 2. Решение 10-го задания, суммарное время решения первых девяти заданий и отклонения от среднего

\begin{tabular}{|l|c|c|c|}
\hline $\begin{array}{c}\text { № } \\
\text { п/п }\end{array}$ & Решение 10-го задания & $\begin{array}{c}\text { Суммарное время } \\
\text { решения первых } \\
\text { девяти заданий, сек }\end{array}$ & $\begin{array}{c}\text { Отклонение суммарного времени } \\
\text { решения первых девяти заданий от } \\
\text { среднего, сек }\end{array}$ \\
\hline 1 & Не решил 10-е задание & 1001,12 & 486,26 \\
\hline 2 & Не решил 10-е задание & 1248,50 & 238,87 \\
\hline 3 & Не решил 10-е задание & 1356,15 & 131,23 \\
\hline 4 & Не решил 10-е задание & 1408,71 & 78,67 \\
\hline 5 & Не решил 10-е задание & 2103,66 & $-616,28$ \\
\hline
\end{tabular}

Из таблицы 1 видно, что быстрота решения первых девяти заданий не предсказывает ни быстроты, ни правильности решения последнего задания. Из пяти не решивших десятое задание (табл.2) двое выполнили первые девять заданий быстрее всех в группе, и у всех, кроме одного время решения первых девяти заданий меньше среднего по группе. 
Кроме этого, видно, что разброс по времени решения последнего задания представляет собой $\mathrm{t}_{10(\max -\min )}=653,22$ секунды, от 374,28 сек до 1027,50 сек, тогда как разброс по доле времени решения последнего задания в суммарном времени решения всех десяти заданий теста представляет собой $\mathrm{d}_{10(\max -\min )}=16 \%$, от $22 \%$ до $38 \%$.

Однако самих по себе этих данных недостаточно для анализа. Ниже будут представлены результаты опроса испытуемых по поводу переживания ими присутствия.

\section{2. Результаты опроса о переживании присутствия в ходе решения теста «Кубики Koca»}

Из 11 решивших последнее задание четверо указали на переживание присутствия в ответе на вопрос «Было ли ощущение, что вы находитесь в том, виртуальном пространстве?». Ещё двое при ответе на этот вопрос указали, что, хотя большую часть времени они не чувствовали, что находятся там, в какой-то момент у них такое чувство было. Ещё в одном случае у испытуемого была потеря представления о положении своего тела (не заметил, как постепенно поворачивался в ходе решения теста.

Из 5 нерешивших у двух было ощущение присутствия, хотя и с оговорками, у одного переживание феномена присутствия было только в самом начале, у остальных такого ощущения не было.

Ниже будут приведены примеры ответов некоторых испытуемых на основные пункты интервью в сочетании с их результатами решения теста. Нумерация сохраняется та же, что была представлена в таблице 1.

Испытуемая 1.

Реальность пространства: нет.

Желание исследовать пространство: нет.

Желание взглянуть на руки: хотела, когда путалась в кнопках.

Желание раздвинуть кубики руками: не хотела.

Посторонние звуки: вопрос не задан.

Присутствие экспериментатора: вопрос не задан.

Время решения 10-го задания: 374,28 сек.

Отклонение от среднего времени решения 10-го задания $(\mathrm{n}=11): 315,72$ сек.

Доля времени решения десятого задания в суммарном времени решения теста: $22 \%$.

Отклонение от средней доли решения десятого задания в суммарном времени решения теста: $9 \%$

Суммарное время решения первых девяти заданий: 1326,52 сек.

Отклонение от среднего значения суммарного времени решения первых девяти задание $(\mathrm{n}=16): 160,86$ сек.

Соотнесение ответов с результатами теста показывает, что испытуемая 1 быстро (с наилучшим результатом в группе) решила задачу, не переживая никаких специфических для виртуальной реальности феноменов.

Испытуемый 4.

Реальность пространства: воспринимал как картинку.

Желание исследовать пространство: нет. Бывало желание запустить кубиком в стену изза рассказов от участника разработки, однако преодолел это ради сосредоточенности на задании.

Желание взглянуть на руки: сначала ответил «нет». Позже добавил: «Мне было интересно, а где мои ноги, где мои руки... в первый раз только когда делал».

Желание раздвинуть кубики руками: нет. Понимал, что управление осуществляется джойстиком.

Другие желания: встать и взглянуть на стол сверху. Не осуществил, потому что до этого понял, что это действие не даёт изменения угла обзора («я до этого попривставал и понял, что это не работает»). 
Посторонние звуки: не слышал; в комнату по мнению испытуемого никто не входил. Это соответствовало действительности.

Присутствие экспериментатора: помнил, ощущал на одном и том же месте относительно себя, но не заметил, что сам сильно развернулся.

Время решения 10-го задания: 578,38 сек.

Отклонение от среднего времени решения 10-го задания $(\mathrm{n}=11): 111,63$ сек.

Доля времени решения десятого задания в суммарном времени решения теста: $33 \%$.

Отклонение от средней доли решения десятого задания в суммарном времени решения теста: $-2 \%$

Суммарное время решения первых девяти заданий: 1199,08 сек.

Отклонение от среднего значения суммарного времени решения первых девяти задание $(\mathrm{n}=16): 288,30$ сек.

Таким образом, можно сказать, что испытуемый 4 в основном не испытывал феномена присутствия. Однако его ответы указывают на некоторые элементы переживания феномена присутствия, например, тот факт, что он не обращал внимания на изменение положения своего тела.

Испытуемая 5.

Ощущение, что находится «там»: было.

Желание исследовать пространство: возникало, но испытуемая ему не следовала, так как считала, что пространство ограничено и, если она повернётся, у неё не получится увидеть то, что находится сзади.

Желание взглянуть на руки: нет.

Желание раздвинуть кубики руками: да.

Другие желания: вопрос не задан.

Посторонние звуки: не слышала. Из наблюдений: прозвучала смс, был шум за окном, кто-то ходил в коридоре и громко разговаривал.

Присутствие экспериментатора: помнила, ощущала на том же месте, где та и сидела. После уточнения, где была экспериментатор, если испытуемая была «там»: «Где-то... (смех) за пределами... пространства с кубиками». После вопроса о конкретном или абстрактном местоположении экспериментатора: «Ну... скорее всего, абстрактное, как... в играх как раз бывает... что... играешь и за кадром голос диктора. Что-то... он диктует, показывает, как надо управлять. Вот так же здесь.»

Время решения 10-го задания: 579,89 сек.

Отклонение от среднего времени решения 10-го задания $(\mathrm{n}=11): 110,12$ сек.

Доля времени решения десятого задания в суммарном времени решения теста: $26 \%$.

Отклонение от средней доли решения десятого задания в суммарном времени решения теста: $4 \%$

Суммарное время решения первых девяти заданий: 1618,34 сек.

Отклонение от среднего значения суммарного времени решения первых девяти задание $(\mathrm{n}=16):-130,96$ сек.

Таким образом, испытуемая 5 показала хорошие результаты (лучше среднего) по решению десятого задания, хотя первые девять она решала медленнее среднего по группе. Она игнорировала внешние стимулы, но при этом помнила о присутствии рядом экспериментатора. Её описание переживаемого ею социального присутствия представляет большой интерес.

Испытуемый 7.

Ощущение, что находится «там»: в основном нет, но «в один момент всё-таки было».

Желание исследовать пространство: было. Следовал ему в то время, когда передвигал курсор на кнопку «следующее задание».

Желание взглянуть на руки: не было. Понимал, что держит геймпад.

Желание раздвинуть кубики руками: не было. 
Другие желания: хотел посмотреть на свои ноги. Напомнил себе, что их не видно, и перестал. Хотел подвигаться, когда устал сидеть, закружилась голова, так как было ощущение, что не сидит, а стоит. Напомнил себе, что сидит, ведь ощущение, что устал сидеть, и головокружение прошло.

Посторонние звуки: слышал. Шуршание, скрип своих ботинок, шаги в коридоре.

Присутствие экспериментатора: непрерывно ощущалось в реальном местоположении.

Время решения 10-го задания: 710,07 сек.

Отклонение от среднего времени решения 10-го задания $(\mathrm{n}=11):-20,06$ сек.

Доля времени решения десятого задания в суммарном времени решения теста: $35 \%$.

Отклонение от средней доли решения десятого задания в суммарном времени решения теста: $-4 \%$

Суммарное время решения первых девяти заданий: 1298,68 сек.

Отклонение от среднего значения суммарного времени решения первых девяти задание $(\mathrm{n}=16): 188,70$ сек.

Здесь интересно то, что об испытуемом 7 можно сказать, что он делал сознательный выбор между переживанием и непереживанием присутствия и останавливал себя, когда начинал ощущать элементы феномена присутствия. По времени решения его результаты немного хуже среднего по последнему заданию и немного лучше среднего по первым девяти.

Испытуемая 11.

Ощущение, что находится «там»: «Ну... скорее нет, чем да. ... чуть-чуть есть, э-э-э... потеря ориентации, вот... но при этом в принципе понимание, я здесь, а оно там».

Желание исследовать пространство: нет. Была занята задачей.

Желание взглянуть на руки: вопрос не задан. В рамках ответа на вопрос про ощущение «там»: «я один раз попыталась посмотреть на джойстик, чтобы посмотреть, где находится нужная мне кнопка, и поняла, что я не могу посмотреть (смех). Вот... и... когда... хотелось... э-э-э... почесать нос, то, конечно, я не сориентировалась, что вообще-то я за столом сижу и рукой вверх держит стол».

Желание раздвинуть кубики руками: нет. «Было очень чёткое ощущение, что я управляю именно... с помощью... джойстика».

Другие желания: вопрос не задан.

Посторонние звуки: слышала, как что-то шумит за окном, дыхание экспериментатора, .

Присутствие экспериментатора: постоянно ощущалось; испытуемая испытывала желание поговорить с экспериментатором.

Время решения 10-го задания: 1027,50 сек.

Отклонение от среднего времени решения 10 -го задания $(\mathrm{n}=11):-337,49$ сек.

Доля времени решения десятого задания в суммарном времени решения теста: 35 \%.

Отклонение от средней доли решения десятого задания в суммарном времени решения теста: $-4 \%$

Суммарное время решения первых девяти заданий: 1895,93 сек.

Отклонение от среднего значения суммарного времени решения первых девяти задание $(\mathrm{n}=16):-408,55$ сек.

Результаты испытуемой 11 заметно хуже средних показателей по группе. При этом она не испытывала пространственного присутствия и испытывала заметное социальное (не только знала, где экспериментатор, но и хотела разговаривать с ней).

Испытуемый 12.

Ощущение, что находится «там»: «Да, было что-то похожее, и я отметил, что, видимо, программа специально написана так, чтобы вот... как бы, чтобы передо мной здесь стол и там тоже стол и чтобы я ощущал как-то... себя там». Ощущение возникало, когда испытуемый отвлекался от решения задачи и исчезало, когда погружался в решение. «М-мМ... ну, тогда (во время сосредоточения на решении) вот я вполне ощущал, что я... сижу. На 
кресле. И что... не перед виртуальным столом. Перед другим столом. И что вот, если я немного поддамся вперёд, я натолкнусь именно на этот стол. Не на виртуальный.»

Желание исследовать пространство: «Да. Я... смотрел вверх, вниз. Отметил, что сверху тоже что-то было. То ли похоже на зеркало, то ли ещё что. Ну, то есть на отражение. Да, в общем... было интересно посмотреть по сторонам». Из ответа на вопрос про ощущение себя там: «Ну, какое-то время, именно когда вот я... когда мне было немного скучно, потому что (неразборчиво) кубик, он двигается медленно и, пока он движется, я мог посмотреть там вниз, верх, оценить обстановку, что там за столом диван, вот. Когда вот я позволял себе так отвлекаться, такое ощущение начиналось».

Желание взглянуть на руки: нет.

Желание раздвинуть кубики руками: «М-м-м... нет, не было. У меня чёткая ассоциация моего присутствия в этом... в этой... М... мире... с исключительно ловушкой, и всё. Были бы у меня там руки, вот, может быть, я бы да, хотел... э-э-э... подвигать своими руками, что ли... что-то ими сделать. Но так нет.».

Другие желания: вопрос не задан.

Посторонние звуки: вопрос не задан.

Присутствие экспериментатора: постоянно ощущалось. «А ещё... да... понял, что я ваше присутствие-то осознавал, ощущал как бы, но... м-м-м... для меня оно в пространстве... перестало быть столь... очевидным... что вы сидите... именно справа. Я просто знал, что где-то вы здесь есть... рядом. Вот. Но точно не слева, точно не сзади, но вот и... не совсем справа.»

Не решил десятое задание.

Суммарное время решения первых девяти заданий: 1001,12 сек.

Отклонение от среднего значения суммарного времени решения первых девяти задание $(\mathrm{n}=16)$ : 486,26 сек.

Испытуемый 12 не решил десятого задания, однако по времени решения первых девяти заданий у него лучший результат в группе. Он испытывал феномен присутствия, но, что интересно, только когда отвлекался от задания. Переживание же им социального присутствия снова демонстрирует тот эффект, когда при переживании пространственного присутствия местоположение «оставшегося в реальности» партнёра по общению становится неясным.

Испытуемый 16.

Реальность пространства: нет.

Желание исследовать пространство: «Ну, я его осмотрел... головой. Картины там, потолок посмотрел».

Желание взглянуть на руки: нет.

Желание раздвинуть кубики руками: нет.

Другие желания: вопрос не задан.

Посторонние звуки: слышал только гул компьютера.

Присутствие экспериментатора: ощущал.

Не решил десятое задание.

Суммарное время решения первых девяти заданий: 2103,66 сек.

Отклонение от среднего значения суммарного времени решения первых девяти задание $(\mathrm{n}=16):-616,28$ сек.

Испытуемый 16 приведён в качестве примера испытуемого, не решившего десятое задание и не испытавшего феномена пространственного присутствия.

\section{4. Заключение}

Данная работа отвечает на поставленные в начале исследования вопросы. Несмотря на некоторую скудость виртуальной среды, используемой при решении интеллектуальной задачи, недостаток событий, происходящих в ней, для изучения присутствия может быть использован 
метод структурированного контекстного интервью. Переживания испытуемых выглядят менее яркими, чем описанные в работе [1], так как в них нет захватывающих событий, таких, как полёт на вертолёте, плавание через озеро, столкновение со стеной и т. п. Однако на вопросы интервью испытуемые давали ответы, раскрывающие их ощущения, могли уточнить свои переживания, реакции и мысли в определённые моменты решения. Испытуемые сообщали как о переживании феномена присутствия на протяжении всего сеанса, так и о частичном. Сообщали они и о некоторых проявлениях, которые принято ассоциировать с переживанием феномена присутствия (не обращали внимания на внешние звуки, на изменения положения собственного тела и т. п.). Кроме того, ответы испытуемых показывали переживание социального присутствия. Поскольку другой человек (экспериментатор) не был представлен в виртуальной среде, можно было ожидать, что социальное присутствие (в данном случае - ощущение, что экспериментатор сидит рядом) будет нарушать переживание пространственного присутствия, однако об этом никогда не сообщалось.

Кроме того, в своих ответах испытуемые показывают интересные эффекты, связанные с представлением о собственном местоположении, местоположении окружающей обстановки и других людей, не представленных в виртуальной реальности (в данном случае экспериментатора). Мысленная структура пространства, в котором испытуемые помещали себя внутрь виртуальной реальности, а экспериментатора - за её пределами, хотя и рядом с собой (что продолжает результаты [1]) заслуживает отдельного изучения. Ответ испытуемого 7, который сознательным усилием «возвращал» себя из переживания феномена присутствия, перекликается с работами группы M. Slater, [14 - 16], в которых переключение между реальным миром и виртуальной средой сравнивается с переключением между фигурой и фоном при просмотре иллюзий на основе гештальта.

Интересно, что некоторые испытуемые показывали, что переживали присутствие, когда отвлекались от задачи или что необходимость сосредотачиваться не давала им осмотреться в среде. Возможно, интеллектуальная деятельность препятствует переживанию феномена пространственного присутствия из-за того, что всё внимание уделяется задаче, а не оформлению среды. Однако для уточнения этого требуются дополнительные исследования.

Пока рано делать однозначные выводы о взаимосвязи решения задачи и переживания феномена присутствия, однако ясно одно: феномен присутствия, пусть и не в очень яркой форме, может быть пережит в бедной событиями и интерактивными объектами среде, предназначенной для решения интеллектуальной задачи.

\section{Литература}

[1] Авербух Н. Особенности переживания феномена присутствия // Информационное общество: образование, наука, культура и технологии будущего. Выпуск 2: Труды XXI Международной объединенной конференции «Интернет и современное общество, IMS-2018 (Санкт-Петербург, 30 мая - 2 июня 2018 г.). Сборник научных статей - СПб: Университет ИТМО. 2018. С. 225 - 239.

[2] Авербух Н.В., Щербинин А.А. Феномен присутствия и его влияние на эффективность решения интеллектуальных задач в средах виртуальной реальности // Психология. Журнал Высшей школы экономики. 2011. Том 8, № 4. С. 102 - 119.

[3] Величковский Б. Б. Влияние обнаружения и коррекции ошибок на феномен присутствия в виртуальных средах // Вестник московского университета. Серия 14. Психология. 2016. № 3. С. 25 - 33.

[4] Величковский Б.Б. Психологические факторы возникновения чувства присутствия в виртуальных средах. // Национальный психологический журнал. 2014. №3(15). С. 31 38.

[5] Величковский Б.Б. и др. Когнитивный контроль и чувство присутствия в виртуальных средах / Величковский Б.Б., Гусев А.Н., Виноградова В.Ф., Арбекова О.А. // Экспериментальная психология. 2016. Т.9. №1. С. 5 - 20. 
[6] van Baren J., IJsselsteijn W. Measuring Presence: A Guide to Current Measurement Approaches. // Deliverable of the OmniPres project IST-2001-39237. 2004.

[7] Garau M. et al. Temporal and Spatial Variations in Presence: A Qualitative Analysis / Garau M., Ritter-Widenfeld H., Antley A., Friedman D., Brogni A., Slater M. // Presence. 7th International Conference on Presence. 2004. Valencia, Spain. P. 232 - 239.

[8] Immersed in Media: Telepresence Theory, Measurement \& Technology / New York, NY: Springer. 2015.

[9] Lombard M., Jones M. T. Defining presence // Immersed in Media: Telepresence Theory, Measurement and Technology. 2015. London: Springer. P. 13 - 34.

[10] Mestre D. R. Presence in Virtual Reality: insights from fundamental and applied research // Electronic Imaging, The Engineering Reality of Virtual Reality. 2018, P. 433-1 - 433-5.

[11] Schwind V. et al. Using Presence Questionnaires in Virtual Reality / Schwind V., Knierim P., Haas N., Henze N. // CHI '19 Proceedings of the 2019 CHI Conference on Human Factors in Computing Systems. Paper No. 360. Glasgow, Scotland Uk - May 04 - 09, 2019.

[12] Skarbez R., Brooks Jr F., Whitton M. A survey of presence and related concepts // ACM Computing Surveys. 2017. Vol. 50(6). P. 1-39.

[13] Slater M. Place illusion and plausibility can lead to realistic behaviour in immersive virtual environments // Philosophical Transaction of Royal Society B: Biological Sciences. 2009. № 364, P. $3549-3557$.

[14] Slater M. Presence and The Sixth Sense // Presence. 2002. Vol. 11, № 4. P. 435 - 439.

[15] Slater, M., Brogni, A., Steed, A. Physiological Responses to Breaks in Presence: A Pilot Study // The 6th Annual International Workshop on Presence. 2003. Aalborg, Denmark.

[16] Slater M., Steed A. A Virtual Presence Counter // Presence: Teleoperators and Virtual Environments. 2000. Vol. 9. Issue 5. P. 413 - 434.

[17] Steed A. et al. "We Wait" - The Impact of Character Responsiveness and Self Embodiment on Presence and Interest in an Immersive News Experience / Steed A, Pan Y, Watson Z., Slater M. // Front. Robot AI 5:112. 2018.

[18] Velichkovsky B. The relationship between interference control and sense of presence in virtual environments // Psychology in Russia: State of the Art. 2017. Vol. 10, № 3. P. 165 176.

[19] Velichkovsky B. B. et al. Cognitive control influences the sense of presence in virtual environments with different immersion levels / Velichkovsky B. B., Gusev A. N., Kremlev A. E., Grigorovich S. S. // Lecture Notes in Computer Science. 2017. Vol. 10324. P. 3 - 16.

[20] Velichkovsky B. B. et al. Error monitoring and correction related to the sense of presence in virtual environments // Velichkovsky B. B., Gusev A. N., Kremlev A. E., Grigorovich S. S. // Communications in Computer and Information Science. 2017. Vol. 714. P. 73 - 80.

[21]Witmer B.G., Singer M.J. Measuring Presence in Virtual Environments: A Presence Questionnaire // Presence. 1998. Vol. 7. № 3. P. 225 - 240.

[22]Zhang C., Perkis A., Arndt S. Spatial Immersion versus Emotional Immersion, Which is More Immersive? // 2017 Ninth International Conference on Quality of Multimedia Experience (QoMEX). 31 May-2 June 2017. DOI: 10.1109/QoMEX.2017.7965655.

\title{
The Presence in Solving Intellectual Tasks: Results of Qualitative Research
}

\author{
N.V. Averbukh
}

Ural Federal University

This paper is devoted to the problem of studying the phenomenon of presence in solving intellectual problems. "Kohs Block Design Test" was selected as the intellectual tasks. The phenomenon of presence is investigated by means of structured contextual interview. 
This paper raises the question of the possibility of studying the presence in solving the intellectual problem in the intended virtual environment, in connection with this poor objects and events.

The results show that the phenomenon of presence can be combined with a successful solution of the intellectual problem, and with the inability to solve it. It is shown how spatial and social presence are experienced in the solution.

Keywords: virtual reality, the phenomenon of presence, spatial presence, social presence, Kohs Block Design Test

Reference for citation: N.V. Averbukh The Presence in Solving Intellectual Tasks: Results of Qualitative Research // Information Society: Education, Science, Culture and Technologies of the Future. Vol. 3 (Proceedings of the XXII International Joint Scientific Conference «Internet and Modern Society», IMS-2019, St. Petersburg, June 19-22, 2019). - St. Petersburg: ITMO University, 2019. P. 211 - 225. DOI: 10.17586/2587-8557-2019-3-211-225

\section{Reference}

[1] Averbukh N. Osobennosti perezhivaniya fenomena prisutstviya [The experience of the phenomenon of presence] // Informacionnoe obshchestvo: obrazovanie, nauka, kul'tura i tekhnologii budushchego. Vypusk 2: Trudy XXI Mezhdunarodnoj ob"edinennoj konferencii «Internet i sovremennoe obshchestvo, IMS-2018 (Sankt-Peterburg, 30 maya - 2 iyunya 2018 g.). Sbornik nauchnyh statej [Information society: education, science, culture and technology of the future. Issue 2. Proceedings of the XXI international joint conference " Internet and modern society, IMS-2018, (St. Petersburg, may 30-June 2, 2018) Collection of scientific articles]. - St. Petersburg: ITMO University, 2018. P. 225 - 239. (In Russian).

[2] Averbukh N.V., Shcherbinin A.A. Fenomen prisutstviya i ego vliyanie na effektivnost' resheniya intellektual'nyh zadach v sredah virtual'noj real'nosti [The Presence Phenomenon and Its Influence upon Intellectual Task Performance within Virtual Reality Settings]. Psihologiya. ZHurnal Vysshej shkoly ekonomiki [The Psychology. Journal of the Higher School of Economics]. V. 8, No 4, 2011. P. 102-119. (In Russian).

[3] Velichkovskij B. B. Vliyanie obnaruzheniya i korrekcii oshibok na fenomen prisutstviya v virtual'nyh sredah [Impact Detection and Error Correction on the Phenomenon of Presence in Virtual Environments] // Vestnik moskovskogo universiteta. Seriya 14. Psihologiya [Moscow University Psychology Bulletin]. 2016. № 3. P. 25 - 33.

[4] Velichkovsky, B.B. Psihologicheskie faktory vozniknoveniya chuvstva prisutstviya v virtual'nyh sredah [Psychological factors of the emerging sense of presence in virtual environments]. // Nacional'nyj psihologicheskij zhurnal [Notional psychological journal] 2014. - №3(15). P. 31-38. (In Russian).

[5] Velichkovskij B.B. et al.. Kognitivnyj kontrol' i chuvstvo prisutstviya v virtual'nyh sredah [Cognitive Control and a Sense of Presence in Virtual Environments] / Velichkovskij B.B., Gusev A.N., Vinogradova V.F., Arbekova O.A. // Eksperimental'naya psihologiya [Experimental Psychology]. 2016. Vol.9. №1. P. 5 - 20.

[6] van Baren J., IJsselsteijn W. Measuring Presence: A Guide to Current Measurement Approaches. // Deliverable of the OmniPres project IST-2001-39237. 2004.

[7] Garau M. et al. Temporal and Spatial Variations in Presence: A Qualitative Analysis / Garau M., Ritter-Widenfeld H., Antley A., Friedman D., Brogni A., Slater M. // Presence. 7th International Conference on Presence. 2004. Valencia, Spain. P. 232 - 239.

[8] Immersed in Media: Telepresence Theory,Measurement \& Technology, eds Lombard M., Biocca F., Freeman J.,Ijsselsteijn W., Schaevitz R.. New York, NY: Springer. 2015.

[9] Lombard M., Jones M. T. Defining presence // Immersed in Media: Telepresence Theory, Measurement and Technology. 2015. London: Springer. P. 13 - 34. 
[10] Mestre D. R. Presence in Virtual Reality: insights from fundamental and applied research // Electronic Imaging, The Engineering Reality of Virtual Reality. 2018, P. 433-1 - 433-5.

[11] Schwind V. et al. Using Presence Questionnaires in Virtual Reality / Schwind V., Knierim P., Haas N., Henze N. // CHI '19 Proceedings of the 2019 CHI Conference on Human Factors in Computing Systems. Paper No. 360. Glasgow, Scotland Uk - May 04 - 09, 2019.

[12] Skarbez R., Brooks Jr F., Whitton M. (2017). A survey of presence and related concepts // ACM Computing Surveys, 50(6), P. $1-39$.

[13] Slater M. Place illusion and plausibility can lead to realistic behaviour in immersive virtual environments // Philosophical Transaction of Royal Society B: Biological Sciences. 2009. № 364, P. 3549 - 3557.

[14] Slater M. Presence and The Sixth Sense // Presence. 2002. Vol. 11, № 4. P. 435 - 439.

[15] Slater, M., Brogni, A., Steed, A. Physiological Responses to Breaks in Presence: A Pilot Study // The 6th Annual International Workshop on Presence. 2003. Aalborg, Denmark.

[16] Slater M., Steed A. A Virtual Presence Counter // Presence: Teleoperators and Virtual Environments. 2000. Vol. 9. Issue 5. P. $413-434$.

[17] Steed A. et al. "We Wait" - The Impact of Character Responsiveness and Self Embodiment on Presence and Interest in an Immersive News Experience / Steed A, Pan Y, Watson Z., Slater M. // Front. Robot AI 5:112. 2018.

[18] Velichkovsky B. The relationship between interference control and sense of presence in virtual environments // Psychology in Russia: State of the Art. 2017. Vol. 10, № 3. P. 165 176.

[19] Velichkovsky B. B. et al. Cognitive control influences the sense of presence in virtual environments with different immersion levels / Velichkovsky B. B., Gusev A. N., Kremlev A. E., Grigorovich S. S. // Lecture Notes in Computer Science. 2017. Vol. 10324. P. 3 - 16.

[20] Velichkovsky B. B. et al. Error monitoring and correction related to the sense of presence in virtual environments / / Velichkovsky B. B., Gusev A. N., Kremlev A. E., Grigorovich S. S. // Communications in Computer and Information Science. 2017. Vol. 714. P. 73 - 80.

[21]Witmer B.G., Singer M.J. Measuring Presence in Virtual Environments: A Presence Questionnaire // Presence. Vol. 7. No 3. 1998. P. 225-240.

[22]Zhang Chenyan, Perkis A., Arndt S. Spatial Immersion versus Emotional Immersion, Which is More Immersive? // 2017 Ninth International Conference on Quality of Multimedia Experience (QoMEX). 31 May-2 June 2017. DOI: 10.1109/QoMEX.2017.7965655. 